\title{
Longitudinal Effects of Medical Comorbidities on Functional Outcome and Life Satisfaction after Traumatic Brain Injury: An Individual Growth Curve Analysis of NIDILIRR Traumatic Brain Injury Model System Data
}

\author{
Dr James F. Malec, PhD, \\ Indiana University School of Medicine, Indianapolis \\ Dr Jessica M. Ketchum, PhD, \\ Craig Hospital, Englewood, Colorado \\ Dr Flora M. Hammond, MD, \\ Indiana University School of Medicine, Indianapolis \\ Dr John D. Corrigan, PhD, \\ The Ohio State University, Columbus \\ Dr. Kristen Dams-O’Connor, \\ Icahn School of Medicine at Mount Sinai, New York, New York \\ Dr Tessa Hart, PhD, \\ Moss Rehabilitation Research Institute, Elkins Park, Pennsylvania \\ Dr Thomas Novack, PhD, \\ The University of Alabama at Birmingham \\ Dr. Marie Dahdah, \\ Baylor Scott \& White Medical Center--Plano, Texas \\ Dr Gale G. Whiteneck, PhD, \\ Craig Hospital, Englewood, Colorado \\ Dr Jennifer Bogner, PhD \\ The Ohio State University, Columbus
}

\begin{abstract}
Objective: To explore associations of specific physical and neuropsychiatric medical conditions to motor and cognitive functioning and life satisfaction over the first 10 years following traumatic brain injury (TBI).
\end{abstract}

Setting: Telephone follow-up through six TBI Model System centers.

Flora M. Hammond, Professor and Chair, Indiana University School of Medicine, 4141 Shore Drive, Indianapolis, IN 46254, UNITED STATES, 317-329-2106, mobile: 317-292-6781, FAX: 317-329-2600, flora.hammond@ rhin.com. 
Participants: 404 individuals or proxies with TBI enrolled in the TBI Model System longitudinal study participating in 10 year follow-up.

Design: Individual growth curve analysis.

Main Measures: FIM ${ }^{\mathrm{TM}}$ Motor and Cognitive subscales, Life Satisfaction Scales, Medical and Mental Health Co-Morbidities Interview.

Results: Hypertension, diabetes, cancers, rheumatoid arthritis, and anxiety negatively affected the trajectory of motor functioning over time. Diabetes, cancers, chronic bronchitis, anxiety, and depression negatively impacted cognitive functioning. Numerous neuropsychiatric conditions (sleep disorder, alcoholism, drug addiction, anxiety, panic attacks, PTSD, depression, bipolar disorder), as well as hypertension, liver disease, and cancers diminished life satisfaction. Other medical conditions had a negative effect on functioning and satisfaction at specific follow-up periods.

Conclusion: Natural recovery after TBI may include delayed onset of functional decline or early recovery followed by progressive deterioration and is negatively affected by medical comorbidities. Results contribute to the growing evidence that TBI is most appropriately treated as a chronic medical condition complicated by a variety of comorbid conditions.

\section{Keywords}

Prevalence; Traumatic Brain Injury; Rehabilitation; Outcomes; Medical Conditions; Comorbidities; Life Satisfaction; Function

\section{INTRODUCION}

There is accumulating evidence that a subset of individuals with traumatic brain injury (TBI) experience functional decline over the years following TBI, while others show improvement or remain stable. ${ }^{1-5}$ A consensus conference of experts in brain injury rehabilitation 6,7 recommended use of the term "chronic brain injury" (CBI) to acknowledge the constellation of persisting TBI symptoms, functional limitations, and secondary health conditions experienced by some survivors of moderate-severe TBI. The World Health Organization defines a chronic disease as one that is permanent, caused by non-reversible pathological alterations, requires specialized rehabilitation, and may require a long period of observation, supervision, or care. ${ }^{8}$ Accordingly, the consensus group further recommended that CBI be considered a chronic health condition that should be proactively monitored and, when indicated, managed as a life-long condition in order to optimize a person's health, independence, and life satisfaction.

Proactive management of TBI as a chronic condition must address any comorbid conditions that are prevalent among individuals with TBI—regardless of whether the condition(s) predate, were caused by, or present in the years subsequent to the TBI. These health comorbidities may combine with TBI-related cognitive, physical, and behavioral sequelae to impact global function and well-being. Research on comorbidities of TBI was summarized in a review by the National Academy of Medicine, which found substantial associations between moderate-severe TBI and a number of psychiatric disturbances and 
neurodegenerative conditions, as well as seizures and premature death. ${ }^{4} \mathrm{~A}$ recent large-scale study of comorbidities at 10 years post moderate-severe TBI confirmed a high prevalence of psychiatric disorders (depression, anxiety, and panic attacks); back pain, hypertension, sleep disorders, and diabetes were also among the most commonly reported conditions. ${ }^{9}$ These findings support a conception of TBI as a chronic health condition that can include progressive deterioration in overall health and functioning.

Almost all prior research on the association between TBI and other medical conditions is cross-sectional so little is known about the association of various co-morbid conditions with the long-term trajectory of function and well-being following TBI. The current study was designed to identify the medical and neuropsychiatric conditions that are associated with functional decline over time, and to examine the associations of each medical condition with the longitudinal trajectory of each outcome (motor functioning, cognitive functioning, and life satisfaction) over time from 1 to 10 years post-injury. This research was largely exploratory since we found little basis in the existing literature for hypotheses about the relationship between specific comorbid conditions and the trajectory of function and wellbeing after TBI.

\section{METHODS}

\section{Participants}

Participants were eligible for this study who met all of the following criteria: (1) previously enrolled in the Traumatic Brain Injury Model Systems (TBIMS) National Database (NDB) at one of 6 participating TBIMS sites; (2) their 10 year follow-up window was due between 7/1/2013 and 9/30/2017; and (3) they had FIM ${ }^{\mathrm{TM}}$ data collected during at least 2 of the three prior follow-up interviews (at 1,2, and 5 years post-injury). All participants included in this study met TBIMS inclusion/exclusion criteria: (1) TBI with the following characteristics (PTA $>24$ hours, or LOC $>30$ minutes, or GCS in $\mathrm{ED}<13$, or intracranial neuroimaging abnormalities), (2) admitted to system's hospital emergency department within 72 hours of injury, (3) 16 years of age or older at the time of injury, (4) receives acute care and comprehensive inpatient rehabilitation within the model system hospitals, and (5) informed consent is signed by patient, family or guardian. Participants were consecutively enrolled in this study when they were contacted for their 10-year telephone follow-up interview. No participant who completed 10-year follow-up refused to participate in the additional data collection for this study. The target sample size was 400. Enrollment was discontinued on 7/20/2016 when an enrollment of 404 participants was reached. Each center's IRB procedures for research with human subjects were followed in conducting this study. As for all TBIMS follow-up interviews, information was obtained from the best source, either the participant with TBI or a significant other. The best source was determined by the TBIMS data collector's protocol to evaluate the participant's cognitive and communication abilities to provide follow-up information. Table 1 provides a more detailed description of sample characteristics. 


\section{Outcome Measures}

FIM $^{\mathrm{TM}}$.- The FIM ${ }^{\mathrm{TM}}$ records functional independence in motor, cognitive, and self-care abilities on a scale from 1 (total dependence) to 7 (complete independence). It has welldocumented metric properties. ${ }^{10,11}$ Rasch analyses revealed two separate domains of items: the motor domain consisting of 13 items and the cognitive domain consisting of 5 items. ${ }^{11,12}$ Subscales for motor (range 13 -91) and cognitive (range 7 - 35) functioning were used in this study. FIM ${ }^{\mathrm{TM}}$ Motor and Cognitive data could be supplied either by the participant or a proxy, and were available for all 404 subjects.

Satisfaction with Life Scale (SWLS).-The SWLS is a 5-item measure of global life satisfaction with established reliability and validity. ${ }^{13,14}$ Higher scores indicate greater satisfaction. This measure was completed only by the person with TBI and could not be completed by a proxy. As a result, the number of cases with SWLS data is less than that for other outcome measures. SWLS data were available for 326 of the 404 subjects $(80.7 \%)$.

\section{Medical and Mental Health Co-Morbidities Interview (MMHCl).-The MMHCI} interview was developed specifically for this study and continues to be used in the TBIMS longitudinal research program. During the study period, some physical medical conditions were queried routinely as part of TBIMS follow-up; others and the mental health conditions were included in the supplemental interview for this project. MMHCI queries about physical medical conditions were modeled after the National Health and Nutrition Examination Survey (NHANES).${ }^{15}$ However, the NHANES survey does not address mental health conditions and was supplemented by mental health items from the National Comorbidity Survey Replication (NCS-R). ${ }^{16}$ All MMCHI queries used the stem "Has a doctor ever told you that you had ...". The MMHCI recorded the extent and chronicity of physical health and mental health comorbidities present across a patient's lifetime, and whether diagnosis occurred before, concurrent with, or after TBI. The person's age at the time the diagnosis was used to determine chronicity. If they had difficulty remembering their age when diagnosed, they were asked how many years ago a doctor told them they had the condition. A complete list of the medical and neuropsychiatric conditions that were queried is available in Hammond et al. ${ }^{9}$

\section{Covariates}

Covariates included sex (male, female), race/ethnicity (white, black, Hispanic, other), marital status (married, not married), level of education (less than high school, high school, some college, bachelors/advanced degree), employment status (employed, i.e., full or parttime paid employment including sheltered or supported work; not employed), age at time of injury, time to first follow commands (in days), FIM ${ }^{\mathrm{TM}}$ Motor at discharge, FIM $^{\mathrm{TM}}$ Cognitive at discharge, and rehabilitation length of stay (LOS). Biologic sex, race/ethnicity, age at time of injury, time to follow commands, discharge FIM data and LOS were obtained from medical record review at time of enrollment into the TBIMS study cohort during inpatient rehabilitation. Extensive description of these variables and methods of collection can be found at www.tbindsc.org. Missing data rates for included covariates were low $(<4 \%)$. Glasgow Coma Scale (GCS) and duration of post-traumatic amnesia (PTA) were not 
included as covariates in the modeling process to avoid multicollinearity issues with time to first follow commands and due to the high rate of missingness (24\%) in GCS.

\section{Procedure}

Participants were asked the supplemental MMCHI questions at the end of the 10-year follow-up interview, which routinely includes completion of the FIM ${ }^{\mathrm{TM}}$ and SWLS. FIM ${ }^{\mathrm{TM}}$ and SWLS were also collected in follow-up interviews 1-, 2-, and 5-year post-injury. Per TBIMS protocol, all follow-up information was collected by telephone interview.

\section{Statistical Analyses}

The prevalence of each of the 44 medical conditions was estimated along with 95\% confidence intervals using the entire sample of 404 participants. Prevalence rates (reported in Hammond et al..$^{9}$ ) were estimated three different ways: (1) for those who have ever been diagnosed with the condition, (2) for those diagnosed with the condition at the same time as or post-TBI, and (3) for those diagnosed with the condition post-TBI. However, because of the scope and complexity of these data, we examined the association between the diagnosis of each medical condition ever (i.e., at any point in time before or after the injury) and the trajectory of function and life satisfaction over a 10-year period in the initial analyses reported here. Missing data on the ever medical conditions ranged from 0 to $1.7 \%$ ( 0 to 7 subjects).

Linear mixed-effects models (specifically, random coefficient models) were used to assess the effect of each medical condition on the longitudinal trajectory of each outcome (FIM ${ }^{\mathrm{TM}}$ Motor, FIM ${ }^{\mathrm{TM}}$ Cognitive, and SWLS) over time from 1 to 10 years post-injury. Mixedeffects models were chosen due to their ability to account for the correlations in the repeated measures from each subject, handle missing data, and flexibility in modeling change over time. SAS v.9.4 ${ }^{17}$ was used for all data analyses. Only subjects with outcome data for at least 3 time points and conditions present in 10 or more subjects were included in these analyses.

The first step was to determine the most appropriate relationship (linear, quadratic, or cubic) between each outcome and time. First, an intercept-only model for each outcome was fit, including a random intercept for each subject. Next, a linear relationship was examined by adding in a linear term and a random linear slope for each subject. Third, a quadratic relationship was assumed by adding a quadratic term and a random quadratic slope for each subject. Finally, a cubic relationship was considered by adding a cubic term; a cubic random effect was not considered since significant variation in the third order polynomial term among the subjects is unlikely and often leads to model convergence issues; also not all subjects had data at all 4 time points necessary to fit a subject-specific cubic relationship. All models assumed an unstructured variance-covariance matrix for the random effects. For each outcome, the most appropriate relationship was identified by selecting the best-fitting model as indicated by the lowest Akaike information criterion (AIC).

The next step was to add the set of demographic/injury-related covariates to the model to explain variance in each parameter of interest. We evaluated the fit of each model and the relationship between these characteristics and the outcomes. The final step was to assess the 
effect of each medical condition on outcome while adjusting for the entire set of demographic/injury characteristics. A separate model was fit for each medical condition. Significance levels were not adjusted for multiple comparisons because of the exploratory nature of this research.

\section{RESULTS \\ FIMTM Motor}

Trajectory shape.-First the unadjusted relationship between FIM ${ }^{\mathrm{TM}}$ Motor scores and time was assessed. A quadratic relationship was selected as it showed the best model fit ( $\mathrm{AIC}=10951.8)$ compared to the intercept $(\mathrm{AIC}=11308.0)$, linear $(\mathrm{AIC}=11082.1)$, and cubic $(\mathrm{AIC}=10958.7)$ relationships. On average, FIM $^{\mathrm{TM}}$ Motor scores were 84.9 at 1 year post TBI, increased to 85.7 at 2 years post TBI, further increased to 86.8 at 5 years post TBI, and then decreased to 84.8 by 10 years post TBI.

Demographic/injury characteristics.-Next, the set of demographic/injury effects was added to the quadratic model. These effects allow for mean shifts (up or down) in the quadratic FIM ${ }^{\mathrm{TM}}$ Motor outcome trajectory based on the values of the covariates. A summary of the adjusted model fit is provided in Table 2. Worse (lower) FIM ${ }^{\mathrm{TM}}$ Motor outcomes post-injury were significantly associated with increased age $(p<0.0001)$, longer time to first follow commands $(p<0.0001)$, and lower FIM ${ }^{\mathrm{TM}}$ Motor scores at discharge from inpatient rehabilitation $(p<0.0001)$. There were no significant relationships between FIM ${ }^{\mathrm{TM}}$ Motor outcomes post-injury and sex $(p=0.84)$, marital status $(p=0.23)$, level of education ( $p=0.18$ ), employment status ( $p=0.73$ ), FIM $^{\mathrm{TM}}$ Cognitive scores at rehabilitation discharge ( $p=.48$ ), or rehabilitation length of stay ( $p=0.08)$. Pairwise comparisons suggested that "other" race/ethnicities had lower FIM ${ }^{\mathrm{TM}}$ Motor scores than both whites $(p=0.0362)$ and blacks ( $p=0.0507)$; however the overall race/ethnicity effect was not significant $(p=0.08)$.

Medical conditions.-Finally, each medical condition and its interaction with the intercept (I), linear (L), and quadratic (Q) trajectory effects were added to the adjusted model to determine whether the shape of the FIM ${ }^{\mathrm{TM}}$ Motor outcome trajectory differed significantly between those with and those without each medical condition. These models indicated that those diagnosed with hypertension (I $p=0.0444$ ), diabetes (I $p=0.0206, \mathrm{~L}$ $p=0.0347$ ), other (than skin) cancer ( $\mathrm{L} p=0.0284$ ), rheumatoid arthritis (I $p=0.0019$ ), and anxiety ( $\mathrm{L} p=0.0141, \mathrm{Q} p=0.0338$ ) each showed significantly different FIM ${ }^{\mathrm{TM}}$ Motor outcome trajectories as compared to those not diagnosed with the condition (see Figure 1).

These models were further used to estimate the mean difference in FIM ${ }^{\mathrm{TM}}$ Motor outcomes between those with and without each medical condition at 1,2,5, and 10 years post-injury. FIM $^{\mathrm{TM}}$ Motor outcomes at 10 years post-injury were significantly worse for participants who had ever been diagnosed with myocardial infarction, other heart conditions, asthma, cataracts, and depression. Differences between these specific diagnoses and worse FIM ${ }^{\mathrm{TM}}$ Motor score were not noted in prior follow-up years with the exception of depression where the difference was observed by year 5. Significant differences in FIM ${ }^{\mathrm{TM}}$ Motor scores between individuals with and without a particular medical condition at any follow-up period 
are displayed in Table 3; results for all conditions at all follow-up periods are available in Supplementary Material.

\section{FIM ${ }^{\mathrm{TM}}$ Cognitive}

Analysis of these data followed the same steps as detailed above for FIM ${ }^{\mathrm{TM}}$ Motor.

Trajectory shape.-The quadratic model showed the best model fit (AIC=8266.9) compared to the intercept $(\mathrm{AIC}=8405.7)$, linear $(\mathrm{AIC}=8333.2)$, and cubic $(\mathrm{AIC}=8274.0)$ models. On average, FIM ${ }^{\mathrm{TM}}$ Cognitive scores are 31.6 at 1 year post TBI, increase to 31.7 at 2 years post TBI, further increase to 31.8 at 5 years post TBI, and then decrease to 31.0 by 10 years post TBI.

Demographic/injury characteristics.-A summary of the model fit is provided in Table 2. Worse (lower) FIM ${ }^{\mathrm{TM}}$ Cognitive outcomes post-injury were significantly associated with increased age $(p<0.0001)$, longer time to first follow commands $(p<0.0001)$, longer rehabilitation stays $(p=0.0101)$, lower FIM ${ }^{\mathrm{TM}}$ Cognitive scores at discharge from inpatient rehabilitation $(p<0.0001)$, and lower levels of education ( $p=0.0149)$. There were no significant relationships between FIM ${ }^{\mathrm{TM}}$ Cognitive outcomes post-injury and sex ( $p=0.17$ ), race/ethnicity $(p=0.69)$, marital status $(p=0.24)$, employment status $(p=0.85)$, or FIM ${ }^{\mathrm{TM}}$ Motor scores at rehabilitation discharge $(p=0.07)$.

Medical conditions. - Those diagnosed with diabetes (I $p=0.0178, \mathrm{~L} p=0.0257, \mathrm{Q}$ $p=0.0184$ ), other cancer ( $\mathrm{L} p=0.0452, \mathrm{Q} p=0.0460$ ), chronic bronchitis (I $p=0.0134$ ), anxiety ( $\mathrm{L} p=0.0173, \mathrm{Q} p=0.0224$ ), and depression ( $\mathrm{L} p=0.0204$ ) each showed a significantly different FIM ${ }^{\mathrm{TM}}$ Cognitive outcome trajectory as compared to those not diagnosed with the condition (see Figure 2).

Cognitive outcome was significantly worse at all four follow-up intervals for those who had been diagnosed with anxiety, PTSD, or depression; there was a similar pattern with chronic bronchitis, with 10 year just missing significance $(p=0.0583)$. Panic attacks were associated with worse outcomes at 2, 5, and 10 years; a similar pattern was seen for rheumatoid arthritis, with the difference at 10 years just missing the nominal significance level ( $p=0.0583$ ). Other conditions associated with worse cognitive outcomes included diabetes (Year 1), obsessive-compulsive disorder (Year 2), hypertension (Year 5), as well as heart conditions (other than myocardial infarction) and back pain (at Year 10). Conditions with significant differences in FIM ${ }^{\mathrm{TM}}$ Cognitive scores between individuals with and without a particular medical condition at any follow-up period are displayed in Table 4; results for all conditions at all follow-up periods are available in Supplementary Material.

\section{SWLS}

Trajectory shape.-The linear model showed the best model fit (AIC $=8140.3)$ compared to the intercept $(\mathrm{AIC}=8141.3)$, quadratic $(\mathrm{AIC}=8147.9)$, and cubic $(\mathrm{AIC}=8153.9)$ models. While the linear model showed the best fit, its AIC was just barely less than that of the intercept model and the linear fixed effect for time was not significant $(p=0.84)$, suggesting relatively stable SWLS scores over time post-injury. On average, SWLS scores were 22.4 at 
1 year post TBI, 22.4 at 2 years post TBI, 22.5 at 5 years post TBI, and 22.5 at 10 years post TBI.

Demographic/injury characteristics.-A summary of the model fit is provided in Table 2. SWLS outcomes post-injury were significantly associated with age $(p=0.0083)$, race/ethnicity ( $p=0.0109)$, level of education $(p=0.0011)$ and marital status $(p=0.0321)$. Specifically, SWLS outcomes were significantly higher for younger age, those having a college level of education as compared to all other education levels, those who were married compared to those not married, and for Hispanics as compared to black or white participants. There were no significant relationships between SWLS post-injury and sex ( $p=0.46$ ), employment status $(p=0.37)$, time to first follow commands $(p=0.50)$, FIM $^{\mathrm{TM}}$ Motor $(p=0.41)$ or Cognitive $(p=0.38)$ scores at discharge, or rehabilitation length of stay $(p=0.62)$.

Medical conditions. - Those diagnosed with hypertension (L $p=0.0226$ ), liver disease (I $p=0.0098, \mathrm{~L} p=0.0053$ ), chronic bronchitis (I $p=0.0229$ ), sleep disorder (I $p=0.0248$ ), back pain (L $p=0.0017$ ), alcoholism (I $p=0.0053$ ), drug addiction (I $p=0.0126$ ), anxiety (I $p=0.0093$ ), panic attacks (I $p=0.0267$ ), post-traumatic stress disorder (I $p=0.0285$ ), depression (I $p<0.0001$ ), and bipolar disorder (I $p=0.0168$ ) each showed a significantly different SWLS outcome trajectory as compared to those not diagnosed with the condition (see Figure 3).

Among the health conditions, some showed maximal associations during the early years after injury that were lessened by year 10, including liver disease and chronic bronchitis. Other conditions had larger associations at 5 and 10 years after injury, perhaps reflecting aging effects, including rheumatoid arthritis, fractures, and back pain. There were several areas of health condition that had significant and persistent associations with life satisfaction across all, or almost all, time points, including sleep disorder, alcoholism, drug addiction, anxiety, panic attacks, PTSD, depression, and bipolar disorder. Conditions with significant differences in SWLS scores between individuals with and without a particular medical condition at any follow-up period are displayed in Table 5; results for all conditions at all follow-up periods are available in Supplementary Material.

\section{Interactive Tool}

We also provide in Supplementary Material a user-friendly interactive tool displaying the FIM Motor, FIM Cognitive, and SWLS trajectories for each medical condition. Users can select specific medical conditions of interest and manipulate the values of the covariates to see estimated trajectories. This manuscript presents only a few of the numerous trajectories, all assumed at the average (or most frequent) values of the covariates.

\section{DISCUSSION}

This study sought to explore the effect of medical and neuropsychiatric co-morbidities on outcomes over the 10 years following acute rehabilitation for TBI. Regardless of the presence of co-morbidities, the overall trajectories of functional abilities over the first 10 years post-injury differed from that of life satisfaction. Motor and cognitive abilities were 
best characterized by a quadratic model, meaning that initial improvement in function was followed by decline. In contrast, life satisfaction was best characterized as a linear trajectory with a relatively flat slope, meaning subjective well-being changed very little across time. The distinct mean trajectory shapes between function and life satisfaction suggest that the latter may be influenced by factors other than change in functional abilities.

The relationships between the outcomes studied and demographic characteristics were consistent with previous studies. Older age at injury was associated with less functional independence and lower life satisfaction. Less education was associated with poorer functional cognitive abilities and life satisfaction, but not motor function. Other demographic characteristics, specifically marital status and Hispanic origin were associated with greater life satisfaction. There were no significant sex differences for the outcomes studied here.

The relationships between outcomes and injury characteristics were also consistent with previous studies. Greater injury severity as captured by time to first follow commands was related to both motor and cognitive function. Discharge motor function was associated with follow-up motor function; a similar pattern was observed for cognitive function, but discharge status for one type of function did not predict the other. Rehabilitation length of stay was associated with long-term cognitive, but not motor, function. Life satisfaction was independent of any injury characteristics, suggesting again that subjective well-being is not determined by injury severity or functional impairment.

The associations of functional trajectories with medical co-morbidities above and beyond injury and acute care characteristics were significant and varied. Persons with cardiac disease, asthma, and cataracts manifested worse motor function at 10 years than persons without these conditions; cardiac disease (other than myocardial infarction) and back pain were related to significantly worse cognitive function. Sleep disorders and back pain were associated with worse life satisfaction at 10 years post-injury. Some medical conditions were unexpectedly associated with better functioning in the first years post-injury, but with diminished motor function by 10 years later (i.e., hypertension, cardiac disease, asthma and cataracts). A similar pattern was evident for diabetes and cognitive function. These trends were not significant at all time points and many differences were relatively small, necessitating caution in interpretation. Further research should attempt to replicate these patterns, ideally with more sensitive and objective standardized functional outcome measures, to provide a more detailed characterization of the relationships between health conditions and functional outcomes after TBI. For example, development of functional decline could be time-based, as in the case of normal aging, or could reflect acceleration or exacerbation of co-morbidities associated with TBI. Changes in health care practice and accessibility over time also may influence the associations between TBI, co-morbidities, and functioning. Future research should also incorporate more detailed information about the timing of onset of health conditions (e.g. through modeling health conditions as timevarying covariates) and the interaction of multiple conditions to better understand the role of disease onset and multi-morbidity in functional outcomes over time. 
One of the most noteworthy findings of this study is the indication that there is a clear and pressing need among survivors of moderate-severe TBI for proactive health management protocols, and that these protocols will need to address both prevention and treatment of neuropsychiatric diseases that onset before, during, and after the TBI. Neuropsychiatric conditions were most associated with diminished cognitive function and life satisfaction at ten years post-injury; however, depression was also related to later reduction in motor function. Anxiety and depression had greater negative associations with cognitive function and life satisfaction than any other co-morbidity, which was also true for depression with motor function. Panic attacks and PTSD were significantly associated with lower cognitive function and life satisfaction. For life satisfaction only, alcoholism and bipolar disorder were associated with lower subjective well-being. The prominence of associations between neuropsychiatric conditions and life satisfaction was striking. However, somewhat surprisingly, neither alcoholism nor drug addiction were associated with worse cognitive outcomes at any time post-injury.

The study has several limitations. Only individuals who survived at least 10 years post injury were included, and it is reasonable to expect that different trajectories and associations may be found in individuals not surviving 10 years. Follow-up assessments were conducted at 1 , 2,5 , and 10 years post-injury, thus precluding the ability to detect changes in trajectory between these time points. While we report statistically significant differences, the extent of their clinical importance was not evaluated. We also did not assess the severity or management of co-morbidities. As application of the Interactive Tool reveals the impact of co-morbidities on functioning varies with covariates and co-morbidity and would probably also vary with other factors, such as, severity and management, which were not assessed. The participants studied here required inpatient rehabilitation for TBI; thus, our findings may not generalize to individuals who incur TBIs that do not necessitate inpatient rehabilitation. Follow-up information is routinely collected over the telephone in the TBIMS research program. Although Bogner and colleagues ${ }^{18}$ confirmed that data collected in this way are generally highly reliable, the potential for bias or distortion in self-report data from individuals with probable cognitive impairment must be recognized. The design we employed did not allow us to determine causal relationships with outcomes, nor were we able to ascertain if comorbid medical conditions interacted with each other to affect outcomes. Significance levels were not adjusted for multiple comparisons because of the exploratory nature of this research. Finally, given the size of the sample used in the current study, parameter estimates may not necessarily generalize to population-based rates.

\section{Conclusion}

The growing evidence that the natural course of TBI may include delayed onset of functional decline, or early recovery followed by progressive deterioration, is not adequately addressed by the health services and resources that are widely available for individuals living with TBI. Results presented here provide additional empirical support for the notion that TBI may be most appropriately treated as a chronic medical condition that may be complicated by other physical and neuropsychiatric conditions. This study provides an initial description of the medical problems that are experienced by survivors of TBI and their relationship to functional independence and life satisfaction in the decade following injury. 
In the long term, gaining such knowledge about medical comorbidities associated with TBI and their relationships to function and well-being is necessary to adequately care for those with chronic brain injury. Further, this knowledge is expected to contribute to the development, implementation, and testing of clinical models for proactively managing TBI as a chronic condition.

\section{Supplementary Material}

Refer to Web version on PubMed Central for supplementary material.

\section{REFERENCES}

1. Hammond FM, Grattan KD, Sasser H, Corrigan JD, Bushnik T, Zafonte RD, et al. Long-term recovery course after traumatic brain injury: a comparison of the functional independence measure and disability rating scale. Journal of Head Trauma Rehabilitation 2001;16(4):318-29. [PubMed: 11461655]

2. Hammond FM, Grattan KD, Sasser H, Corrigan JD, Rosenthal M, Bushnik T, et al. Five years after traumatic brain injury: a study of individual outcomes and predictors of change in function. Neurorehabilitation 2004;19(1):25-35. [PubMed: 14988585]

3. Hammond FM, Hart T, Bushnik T, Corrigan JD, Sasser H, Hammond FM, et al. Change and predictors of change in communication, cognition, and social function between 1 and 5 years after traumatic brain injury. Journal of Head Trauma Rehabilitation 2004;19(4): 314-28. [PubMed: 15263859]

4. IOM (Institute of Medicine). Gulf War and health, volume 7: Long-term consequences of traumatic brain injury. Washington DC: The National Academies Press; 2009.

5. Masel BE, DeWitt DS. Traumatic brain injury: a disease process, not an event. Journal of Neurotrauma 2010;27(8):1529-40. [PubMed: 20504161]

6. Malec JF, Hammond FM (eds). Brain injury as a chronic condition. Brain Injury Professional 2013;10.

7. Malec JF, Hammond FM, Flanagan S, Kean J, Sander A, Sherer M, Masel BE. Recommendations from the 2013 Galveston Brain Injury Conference for implementation of a chronic care model in brain injury. J Head Trauma Rehabil 2013;28:476-83. [PubMed: 24189287]

8. World Health Organization. Innovative care for chronic conditions: Building blocks for action: Global Report. World Health Organization, 2002.

9. Hammond FM, Corrigan JD, Ketchum JM, Malec JF, Dams-O'Conner K, Hart T, Novack TA, Bogner J, Dahdah MN, Whiteneck GG. Prevalence of medical and psychiatric comorbidities following traumatic brain injury. J Head Trauma Rehabil, under review.

10. Granger CV, Hamilton BB, Linacre JM, Heinemann AW, Wright BD. Performance profiles of the Functional Independence Measure. Arch Phys Rehabil 1993;72:84-89.

11. Heinemann AW, Linacre JM, Wright BD, Granger CV. Relationships between impairment and physical disability as measured by the Functional Independence Measure. Arch Phys Rehabil 1993; 74:566-573.

12. Linacre JM, Heinemann AW, Wright BD, Granger CV, Hamilton BB. The structure and stability of the Functional Independence Measure. Arch Phys Rehabil 1994;75:127-132.

13. Pavot W, Diener E. (1993). Review of the Satisfaction With Life Scale. Psychol Assess 1993;5(2): 164-72.

14. Corrigan J, Bogner J, Mysiw W, Fugate L, Clinchot D. Predicting subjective well-being following traumatic brain injury. Poster presented at "TBI in the 21 st Century: Learning from Models of Research and Service Delivery; Washington, DC, 1999.

15. National Health and Nutrition Examination Survey (NHANES). Center for Disease Control, National Center for Health Statistics. https://www.cdc.gov/nchs/nhanes/index.htm. Accessed 7/9/17. 
16. Kessler RC, Merikangas KR. The National Comorbidity Survey Replication (NCS-R): background and aims. International Journal of Methods in Psychiatric Research 2004;13:60-8. [PubMed: 15297904]

17. SAS. Copyright (c) 2002-2012 by SAS Institute Inc., Cary, NC, USA.

18. Bogner JA, Whiteneck GG, MacDonald J, Juengst SB, Brown AW, Philippus AM, Marwitz JH, Lengenfelder J, Mellick D, Arenth P, Corrigan JD. Test-retest reliability of traumatic brain injury outcome measures: A Traumatic Brain Injury Model Systems study. J Head Trauma Rehabil. 2017;32:E1-E16. doi: 10.1097/HTR.0000000000000291. 


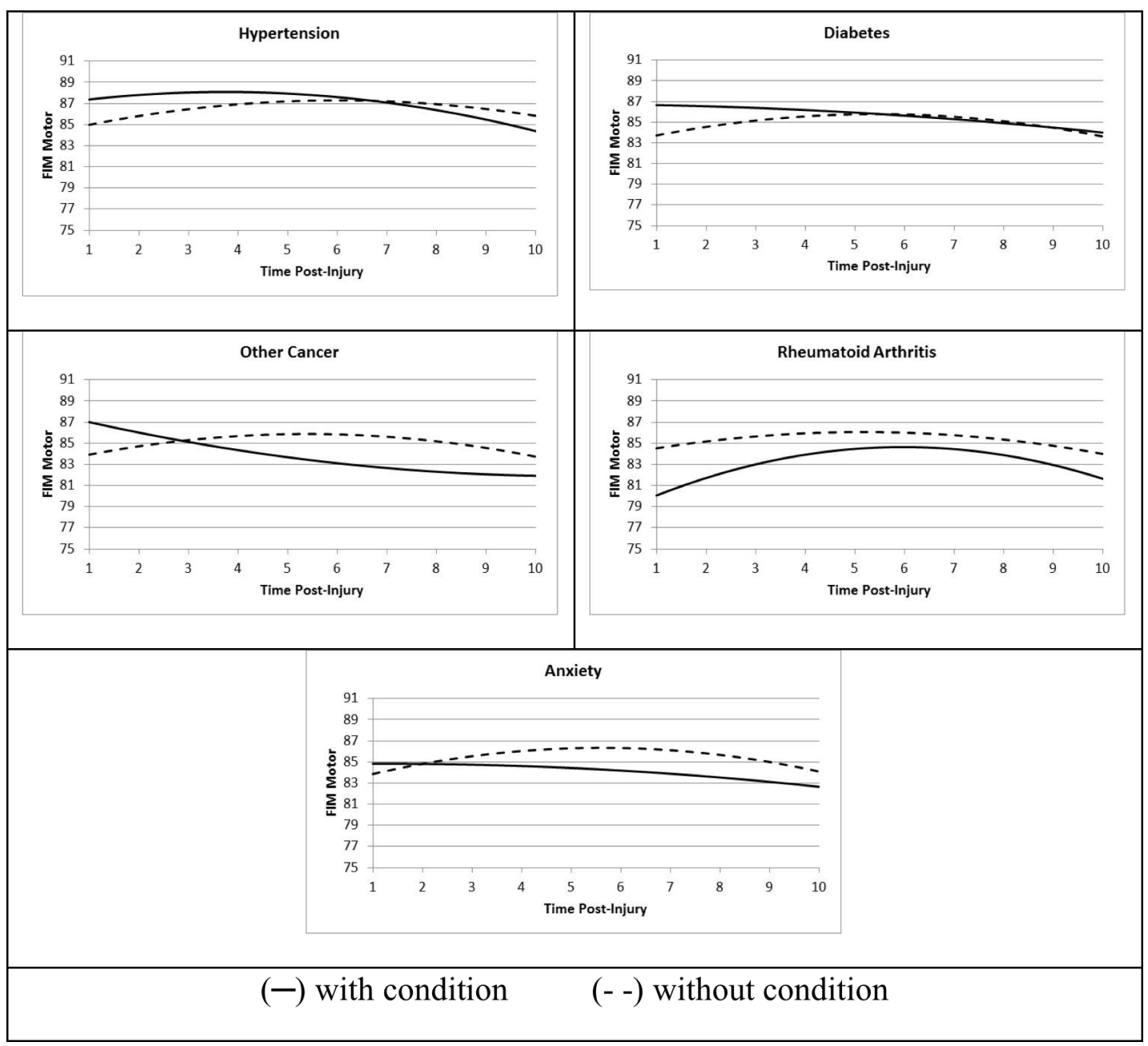

Figure 1: Significantly Different FIM ${ }^{\mathrm{TM}}$ Motor Outcome Trajectories

For Tables 1-3, fixed covariates are male, white, not married, less than HS, employed, age = $37.5, \mathrm{PTA}=31.2, \mathrm{TFC}=10.6, \mathrm{FIM}^{\mathrm{TM}}$ motor discharge $=69.6, \mathrm{FIM}^{\mathrm{TM}}$ cognitive discharge $=$ 23.8, rehabilitation $\mathrm{LOS}=26.0$. 


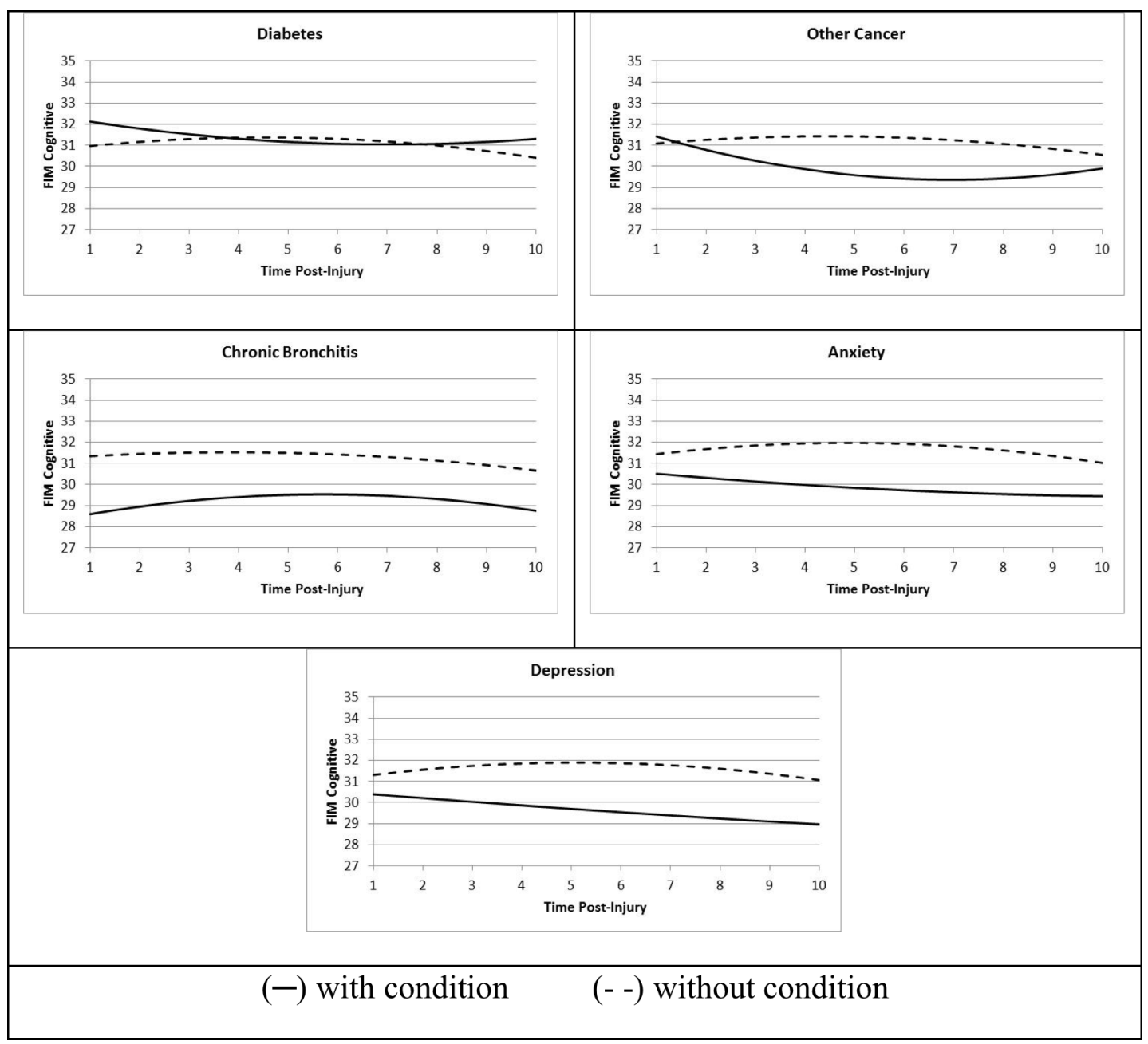

Figure 2:

Significantly Different FIM ${ }^{\mathrm{TM}}$ Cognitive Outcome Trajectories 


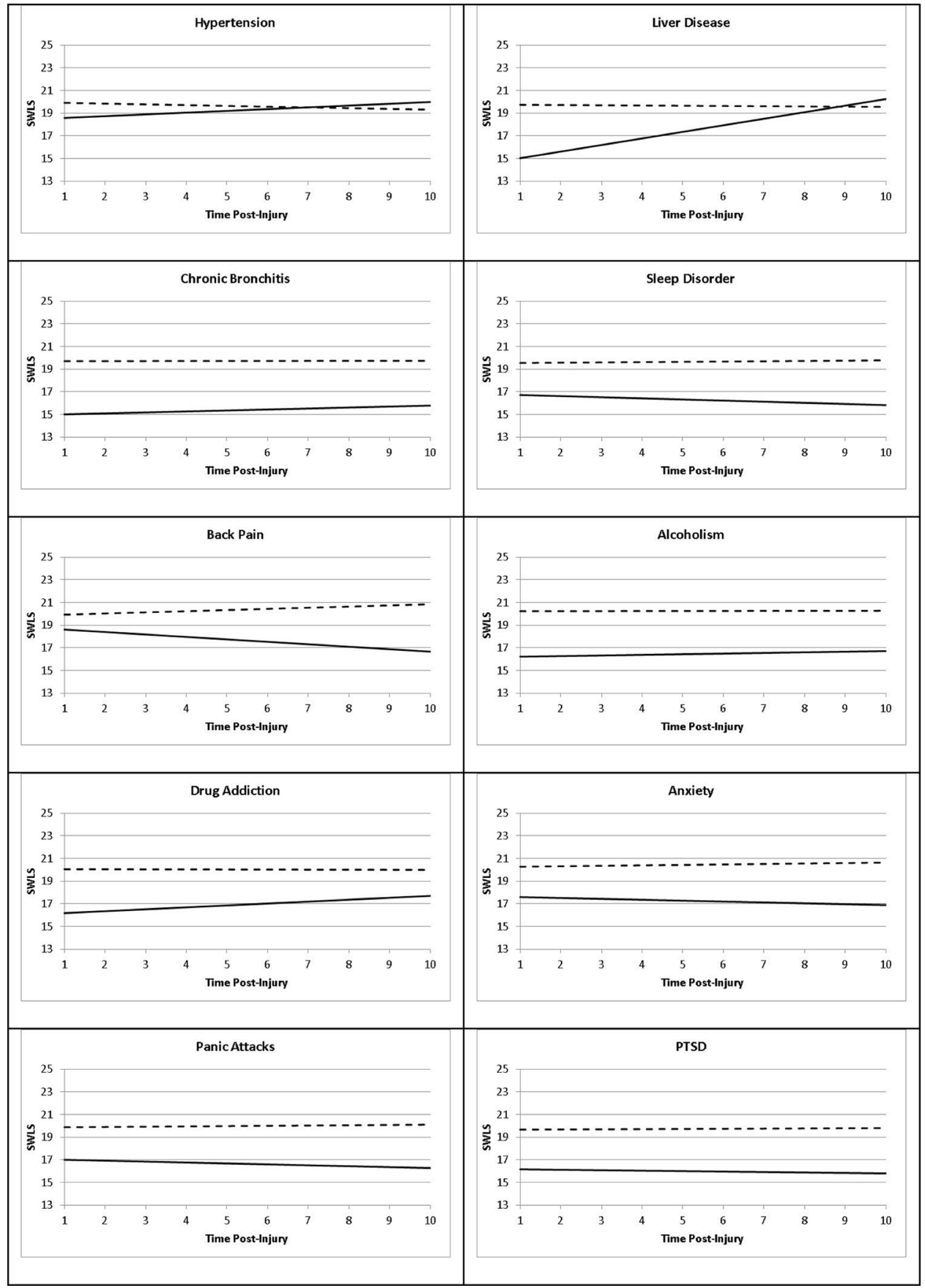

Figure 3:

Significantly Different SWLS Outcome Trajectories

J Head Trauma Rehabil. Author manuscript; available in PMC 2020 September 01. 
Table 1:

Summary of Sample Characteristics $(N=404)$

\begin{tabular}{|c|c|c|c|}
\hline & $N$ & Median (IQR) & Mean (SD) \\
\hline Age at Injury & 404 & $37(23-49.5)$ & $37.8(16.0)$ \\
\hline Age at 10 Year Follow-Up & 404 & $48(33-59)$ & $47.9(15.9)$ \\
\hline GCS & 304 & $9(5-14)$ & $9.5(4.7)$ \\
\hline PTA (days) & 388 & $23(10.5-44)$ & $31.7(31.7)$ \\
\hline Time to First Follow Commands (days) & 400 & $3(0.5-12)$ & $10.5(20.2)$ \\
\hline FIM $^{\mathrm{TM}}$ Motor Discharge & 403 & $72(60-83)$ & $69.2(18.1)$ \\
\hline FIM $^{\mathrm{TM}}$ Cognitive at Discharge & 403 & $25(20-28)$ & $23.5(6.6)$ \\
\hline \multirow[t]{2}{*}{ Rehab LOS (days) } & 404 & $21(13-33)$ & $26.1(19.7)$ \\
\hline & & $N(\%)$ & \\
\hline Sex & 404 & & \\
\hline Female & & $97(24.0 \%)$ & \\
\hline Male & & $307(76.0 \%)$ & \\
\hline Race & 404 & & \\
\hline White & & $280(69.3 \%)$ & \\
\hline Black & & $78(19.3 \%)$ & \\
\hline Hispanic & & $31(7.7 \%)$ & \\
\hline Other & & $15(3.7 \%)$ & \\
\hline Marital Status & 404 & & \\
\hline Married & & $141(34.9 \%)$ & \\
\hline Not Married & & $263(65.1 \%)$ & \\
\hline Education Level & 404 & & \\
\hline Less than HS & & $138(34.2 \%)$ & \\
\hline HS & & $109(27.0 \%)$ & \\
\hline Some College & & $91(22.5 \%)$ & \\
\hline College & & $66(16.3 \%)$ & \\
\hline Employment & 404 & & \\
\hline Employed & & $277(68.6 \%)$ & \\
\hline Not Employed & & $127(31.4 \%)$ & \\
\hline
\end{tabular}

$\mathrm{IQR}=$ interquartile range; $\mathrm{SD}=$ standard deviation; median/IQR preferred over mean/SD as measures of center and spread for skewed variables (PTA, time to first follow commands, rehabilitation LOS) 
Table 2:

Summary of Conditional Models for FIM ${ }^{\mathrm{TM}}$ and SWLS Outcomes

\begin{tabular}{|c|c|c|c|c|c|c|}
\hline & FIM $^{\mathrm{TM}}$ Motor & & FIM ${ }^{\mathrm{TM}}$ Cognitive & & SWLS & \\
\hline Sample Size $(N)$ & 398 & & 398 & & 323 & \\
\hline AIC & 10440.2 & & 7928.4 & & 8031.5 & \\
\hline Random Time Effects & Estimate (SE) & & Estimate (SE) & & Estimate (SE) & \\
\hline Variance (Intercept) & $131.48(12.16)$ & $\dagger$ & $15.86(1.76)$ & $\dagger$ & $39.46(4.67)$ & + \\
\hline Variance (Slope) & $7.51(1.23)$ & + & $2.46(0.35)$ & $\dagger$ & $0.16(0.06)$ & + \\
\hline Variance $\left(\right.$ Slope $\left.^{2}\right)$ & $0.04(0.01)$ & + & $0.01(0.002)$ & + & & \\
\hline Covariance (Intercept, Slope) & $-26.99(3.42)$ & 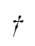 & $-4.61(0.70)$ & $t$ & $-0.62(0.41)$ & \\
\hline Covariance (Intercept, Slope ${ }^{2}$ ) & $1.70(0.26)$ & $t$ & $0.33(0.06)$ & $\dagger$ & & \\
\hline Covariance (Slope, Slope ${ }^{2}$ ) & $-0.48(0.10)$ & + & $-0.19(0.03)$ & + & & \\
\hline Residual & $15.33(1.13)$ & + & $3.47(0.26)$ & $\dagger$ & $24.21(1.42)$ & $\dagger$ \\
\hline Fixed Time Effects & Estimate (SE) & & Estimate (SE) & & Estimate (SE) & \\
\hline Intercept & $80.33(2.89)$ & $t$ & $30.50(1.13)$ & 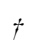 & $24.68(3.20)$ & $t$ \\
\hline Linear Time & $0.98(0.20)$ & $t$ & $0.20(0.10)$ & & $0.01(0.05)$ & \\
\hline Quadratic Time & $-0.09(0.02)$ & $\dagger$ & $-0.02(0.01)$ & $t$ & & \\
\hline Fixed Covariates & $p$-value & & $p$-value & & $p$-value & \\
\hline Sex & 0.8420 & & 0.1669 & & 0.4595 & \\
\hline Race & 0.0839 & & 0.6943 & & 0.0109 & + \\
\hline Marital Status & 0.2283 & & 0.2409 & & 0.0321 & + \\
\hline Education Level & 0.1827 & & 0.0149 & $t$ & 0.0011 & $t$ \\
\hline Employment Status & 0.7281 & & 0.8548 & & 0.3730 & \\
\hline Age at Injury & $<0.0001$ & + & $<0.0001$ & $\dagger$ & 0.0083 & + \\
\hline Time to First Follow Commands & $<0.0001$ & + & $<0.0001$ & 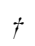 & 0.5016 & \\
\hline FIM $^{\mathrm{TM}}$ Motor Discharge & $<0.0001$ & $t$ & 0.0656 & & 0.4067 & \\
\hline FIM $^{\mathrm{TM}}$ Cognitive Discharge & 0.4840 & & $<0.0001$ & $t$ & 0.3842 & \\
\hline Rehabilitation LOS & 0.0829 & & 0.0101 & $t$ & 0.6225 & \\
\hline
\end{tabular}

$\mathrm{SE}=$ standard error;

${ }_{\text {indicates }} p$-value $<0.05$ 
Table 3:

Statistically significant FIM ${ }^{\mathrm{TM}}$ Motor score differences between participants with and without specific medical diagnoses over 10 year follow-up

\begin{tabular}{lllll} 
Medical Condition & Year 1 & Year 2 & Year 5 & Year 10 \\
\hline Hypertension & $-2.4^{*}$ & $-2.0^{*}$ & -0.7 & 1.5 \\
Myocardial Infarction & -2.2 & -1.2 & 1.9 & $7.7^{* *}$ \\
Other Heart Conditions & -0.9 & -0.8 & 0.3 & $4.7^{* *}$ \\
Asthma & -0.6 & -0.2 & 1.2 & $4.3^{*}$ \\
Diabetes & $-3.5^{*}$ & -2.5 & -0.6 & -1.1 \\
Rheumatoid Arthritis & $4.8^{*}$ & $3.7^{*}$ & 1.9 & 2.7 \\
Cataracts & -1.2 & -1.3 & -0.5 & $4.3 *$ \\
Anxiety & -0.5 & 0.4 & $2.2^{*}$ & 2.0 \\
PTSD & 1.4 & 2.2 & $3.6^{*}$ & 2.9 \\
Depression & 0.7 & 1.2 & $2.4^{* *}$ & $3.4^{* *}$ \\
\hline Positive numbers indicate better function for those without the condition \\
*
\end{tabular}


Table 4:

Statistically significant FIM ${ }^{\mathrm{TM}}$ Cognitive score differences between participants with and without specific medical diagnoses over 10-year follow-up

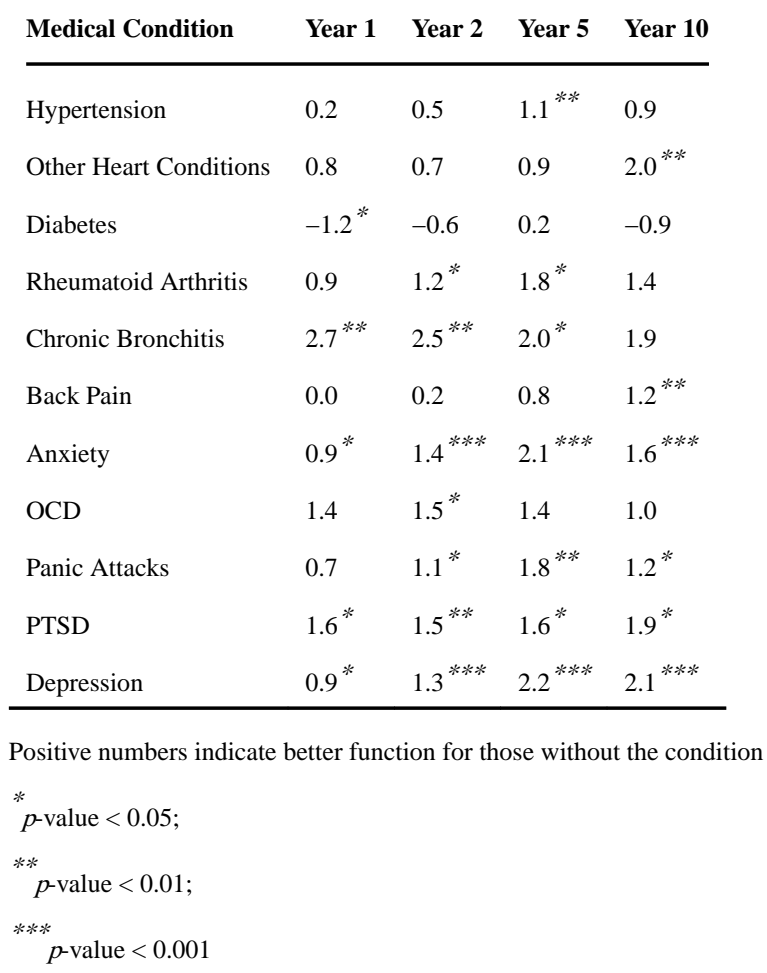


Table 5:

Statistically significant Satisfaction with Life Scale score differences between participants with and without specific medical diagnoses over 10-year follow-up

\begin{tabular}{lllll} 
Medical Condition & Year 1 & Year 2 & Year 5 & Year 10 \\
\hline Liver Disease & $4.7^{*}$ & $4.1^{*}$ & 2.3 & -0.7 \\
Rheumatoid Arthritis & 2.6 & $2.8^{*}$ & $3.3^{*}$ & $4.3^{*}$ \\
Chronic Bronchitis & $4.7^{*}$ & $4.6^{*}$ & $4.4^{*}$ & 4.0 \\
Sleep Disorder & $2.8^{*}$ & $3.0^{* *}$ & $3.3^{* *}$ & $3.9^{* *}$ \\
Fractures & 1.7 & $1.8^{*}$ & $2.1^{*}$ & $2.5^{*}$ \\
Back Pain & 1.3 & 1.6 & $2.6^{* *}$ & $4.2^{* * *}$ \\
Alcoholism & $4.0^{* *}$ & $4.0^{* *}$ & $3.8^{* *}$ & $3.6^{*}$ \\
Drug Addiction & $3.9^{*}$ & $3.7^{*}$ & $3.2^{*}$ & 2.3 \\
Anxiety & $2.7^{* *}$ & $2.8^{* *}$ & $3.2^{* * *}$ & $3.8^{* * *}$ \\
Panic Attacks & $2.9^{*}$ & $3.0^{* *}$ & $3.3^{* *}$ & $3.8^{* *}$ \\
PTSD & $3.5^{*}$ & $3.6^{*}$ & $3.7^{* *}$ & $4.0^{*}$ \\
Depression & $4.7^{* * *}$ & $4.7^{* * *}$ & $4.9^{* * *}$ & $5.1^{* * *}$ \\
Bipolar Disorder & $3.5^{*}$ & $3.5^{* *}$ & $3.5^{* *}$ & $3.6^{*}$ \\
\hline
\end{tabular}

Positive numbers indicate greater satisfaction for those without the condition

*

$p$-value $<0.05$;

**value $<0.01$;

****

$p$-value $<0.001$ 